\title{
NEAR-WALL VELOCITY OF SUSPENDED PARTICLES IN MICROCHANNEL FLOW
}

\author{
Winky L. W. Hau ${ }^{1}$, Zhenyu Liu' ${ }^{2}$, Jan Korvink ${ }^{2}$, Roland Zengerle ${ }^{1,3}$, and Jens Ducrée $e^{1,3}$ \\ ${ }^{1}$ Laboratory for MEMS Applications, Department of Microsystems Engineering (IMTEK), \\ University of Freiburg, Georges-Koehler-Allee 106, D-79110 Freiburg, Germany \\ ${ }^{2}$ Laboratory for Simulation, Department of Microsystems Engineering (IMTEK), University of Freiburg, \\ Georges-Koehler-Allee 106, D-79110 Freiburg, Germany \\ ${ }^{3}$ HSG-IMIT, Wilhelm-Schickard-Straße 10, D-78052 Villingen-Schwenningen, Germany
}

\begin{abstract}
This contribution investigates the characteristic reduction of the particle velocity with respect to the velocity profile of a pure liquid (water) in a pressure driven flow (PDF). It is shown by simulations and experiments that particles are slowed down once their local perturbation "cloud" of the velocity field hits the wall. We show that this effect scales with the ratio of the distance of sphere's surface from the wall, $\delta$, and the radius, $a$, of the sphere, i.e. $\delta / a$.
\end{abstract}

\section{INTRODUCTION}

The motion of a sphere is governed by the hydrodynamic drag from the suspending liquid and its isotropic Brownian movement [1]. These effects are the basis for previous work on (open-tubular) hydrodynamic chromatography (HDC) [2,3]. In HDC, the mean velocity of particles increases with their size as their centers of mass are increasingly excluded from the slow, no-slip region near the wall. Therefore, larger particles move faster than smaller particles.

Instead of a statistical average over an ensemble, we investigated the translational motion of an individual particle. We discovered that the near-wall velocities of particles decrease with their sizes, given their same distances close to the wall. This secondary effect counteracts, to a certain extent, the elevated mean velocity of larger particles in HDC. In this paper, we first set up a physical model by making an analogy. Then we employ an analytical model to benchmark numerical models. Finally, to check the validity of our physical model, we compare the results from both simulations and experiments.

\section{THEORY}

\section{Physical Model}

The presence of a sphere of radius $a$ perturbs the velocity field $u(\mathrm{z})$ of the surrounding liquid (water) layer of thickness $d$ (Figure 1). The thickness $d$ grows with $a$ and we roughly estimate $d \approx a$. The presence of the sphere tends to homogenize the velocity field of the surrounding liquid; hence, the velocity field between the sphere and the wall is elevated with respect to the PDF of a pure liquid.

We now look at two particles positioned at $z=z_{a}$ in the schematics illustrated Figure 1. For the large particle, $\delta \leq d$, the "cloud" hits the wall, i.e. the no-slip boundary condition is partially transmitted via a "cloud" to the sphere, thus tending to reduce the translational velocity of sphere, $U_{a}$. On the contrary, the small particle fulfilling $\delta>d$ is not affected by the additional "friction" with the no-slip region. Its velocity is thus higher than the large particle at the same center of mass position $z=z_{a}$.

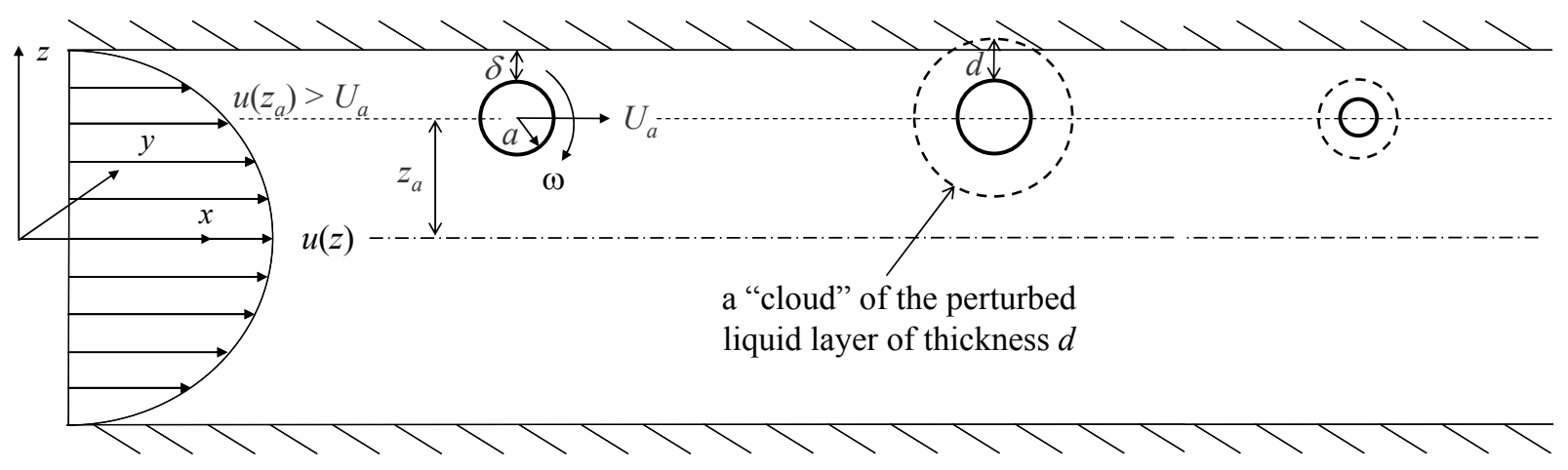

Figure 1: Spheres are transported by a parabolic profile of a PDF between two infinite parallel planes. 


\section{Analytical Model for Particle-Wall Interaction}

The theoretical treatment of a suspended particle in a low Reynolds number flow was developed several decades ago [4]. The derivation is based on the Stokes equation (1) (creeping flow) and the continuity equation (2):

$$
\begin{aligned}
-\nabla p+\mu \nabla^{2} \mathbf{u} & =0 \\
\nabla \cdot \mathbf{u} & =0
\end{aligned}
$$

where $p$ is dynamic pressure, $\mu$ is dynamic viscosity and $\mathbf{u}$ is velocity vector.

The hydrodynamic interaction between the surface of the sphere and the wall was analytically modeled by the so-called "method of reflections". Due to the linearity of the governing equations and the boundary conditions on the sphere and the wall, a systematic scheme of iterations based on superposition was implemented. It allowed the boundary-value problem to be solved to any degree of approximation by considering boundary conditions associated with the sphere or the wall at a time. As shown in Figure 2, this 3-dimensional model quantifies the drag force of a sphere moving at a constant speed in parallel to the two planes. Due to the point-force assumption $[5,6]$, it is valid when the radius of the sphere is much smaller than the distance between its center and the wall, i.e. $a /(a+\delta)$ $<<1$. This analytical model is hence not able to yield our experimental results. However, it helps us to benchmark our finite element models which will be used later in this study to simulate the experimental conditions.

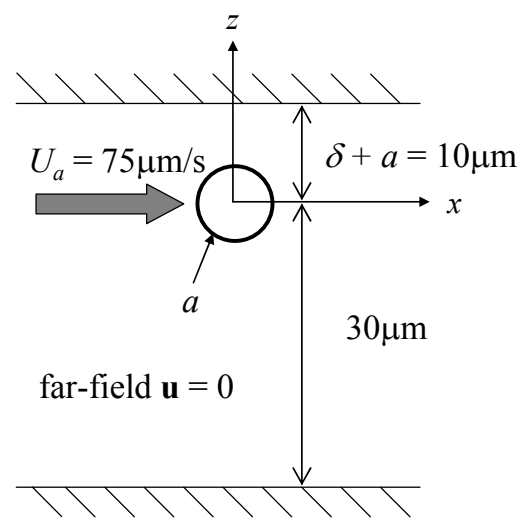

Figure 2: Sketch of the 3D analytical model for a sphere moving in parallel to two planes.

\section{SIMULATIONS}

\section{Benchmarking}

A commercial code (COMSOL Multiphysics 3.3a, Comsol, Sweden) was employed to construct, simulate and post-process the finite element models for benchmarking and simulating the experiment. The typical degree of freedom (DOF) of these numerical models was about 25,000 . A Windows PC with 2GB RAM could process the numerical codes. This is a suspension flow multiphysics simulation - a liquid flow coupled with a rigid body motion. Apart from the Stokes equation and the continuity equation, two more equations were required to simulate the rigid body motion of the sphere, namely translational motion equation and angular motion equation. The boundary condition applied to the sphere's surface was no-slip condition. It was also assumed that the density of the sphere is the same as that of the water; therefore, there was no buoyancy force on the sphere.

In this benchmark model, the drag force from the liquid on the sphere should be constant in a steady state. The results in Table 1 show that the analytical and the numerical models well agree for small ratios $a /(a+\delta)$.

\begin{tabular}{|l|c|c|c|c|}
\hline & \multicolumn{4}{|c|}{ Drag Force |F| on the Sphere } \\
\hline Sphere radius & $(a /(\delta+a))^{2}$ & Analytical & Numerical & Diff. \\
\hline$a=0.91 \mu \mathrm{m}$ & 0.008 & $1.33 \mathrm{pN}$ & $1.36 \mathrm{pN}$ & $0.8 \%$ \\
\hline$a=2.74 \mu \mathrm{m}$ & 0.075 & $4.71 \mathrm{pN}$ & $4.26 \mathrm{pN}$ & $9.6 \%$ \\
\hline
\end{tabular}

Table 1: Results from benchmarking.

\section{Simulating the Experiments}

Based on the numerical simulation already benchmarked, a series of models with two different sizes of $a(0.91 \mu \mathrm{m}$ and $2.74 \mu \mathrm{m}$ ) and several different $\delta$ were built and simulated, the results of which are presented in the next section. Instead of imposing a constant translational velocity to the sphere, a pressure difference was used as boundary condition to drive the liquid flow and thus, the sphere was naturally conveyed by the flow. In this case, the drag force from the liquid on the sphere was zero under a steady state condition.

It should be noted that all dimensionless variables in this paper are labeled with ${ }^{*}$. The velocity of the flow, $u$, and the translational velocity of the sphere, $U_{a}$, are non-dimensionalized by the maximum velocity in the original unperturbed profile while the sphere's position, $z$, the sphere's radius, $a$, the distance, $\delta$, and the thickness, $d$, are non-dimensionalized by one-half of the channel height.

In Figure 3, a dimensionless velocity profile is plotted, annotated with sketches of the sphere, its "cloud" and the wall in proportion. The thickness of the "cloud" $d^{*}$ is proposed to characterize the particle-wall interaction. From the plot, we confirm the above assumption $d^{*} \approx a^{*}$. Consequently, when the "cloud" touches the wall, i.e. for $\delta^{*} \leq d^{*}=a^{*}$, hydrodynamic interaction occurs, resulting in a reduced velocity of the sphere.

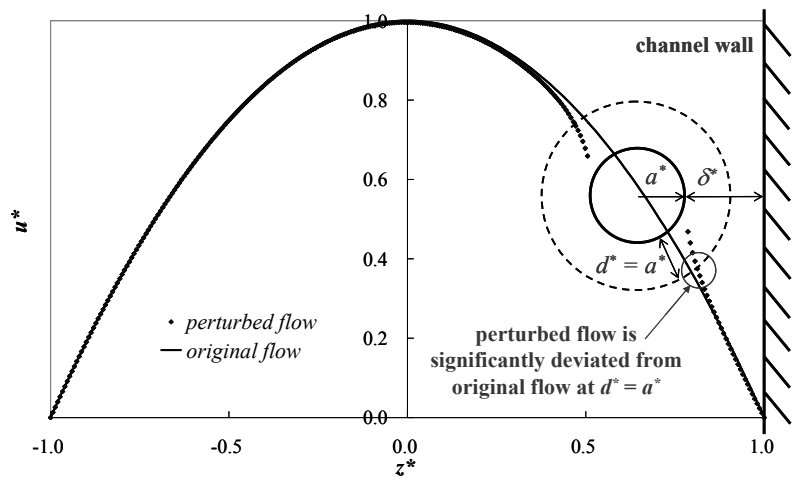

Figure 3: Simulation results revealed the "thickness" $d^{*}$ of the perturbed flow roughly equals the particle radius $a^{*}$. 


\section{EXPERIMENTS}

\section{Experimental Design}

To resemble the infinitely parallel walls and 2D flow conditions, a high-aspect ratio microchannel was used. Polystyrene (PS) particles (microparticles, Germany), with $a$ $=0.91$ and $2.74 \mu \mathrm{m}$, respectively, and density of $1.05 \mathrm{kgm}^{-3}$ were used. The working fluid, deionized (DI) water, was driven by a pressure head between two burettes $(50 \mathrm{ml})$ connected via silicone tubing to the ends of the channel. The typical water column height to produce the pressure head was around $10-30 \mathrm{~mm}$.

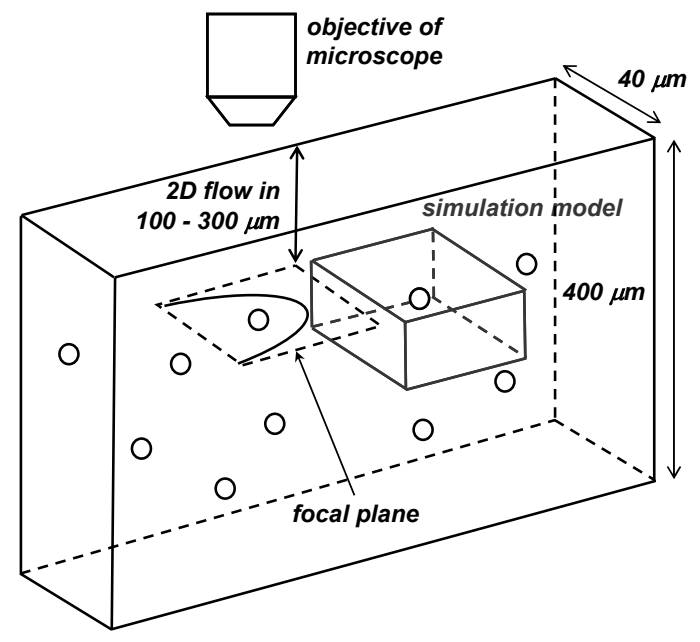

Figure 4: Schematic of the experimental method. The box portion represents the boundaries of the numerical model.

We can observe the motion of spheres whose depth, between $100-300 \mu \mathrm{m}$, coincides with the focal plane (Figure 4). The microchannel measures a width of $40 \mu \mathrm{m}$, a height of $400 \mu \mathrm{m}$ and a length of $70 \mathrm{~mm}$. It was fabricated by a DRIE process on a $100 \mathrm{~mm}$ diameter silicon substrate. The channel was anodically bonded to a Pyrex wafer of the same size exhibiting ultrasonically-drilled fluidic access ports (Figure 5). Finally, two plastic connectors were bonded to the Pyrex cover to provide interconnection for the tubing.

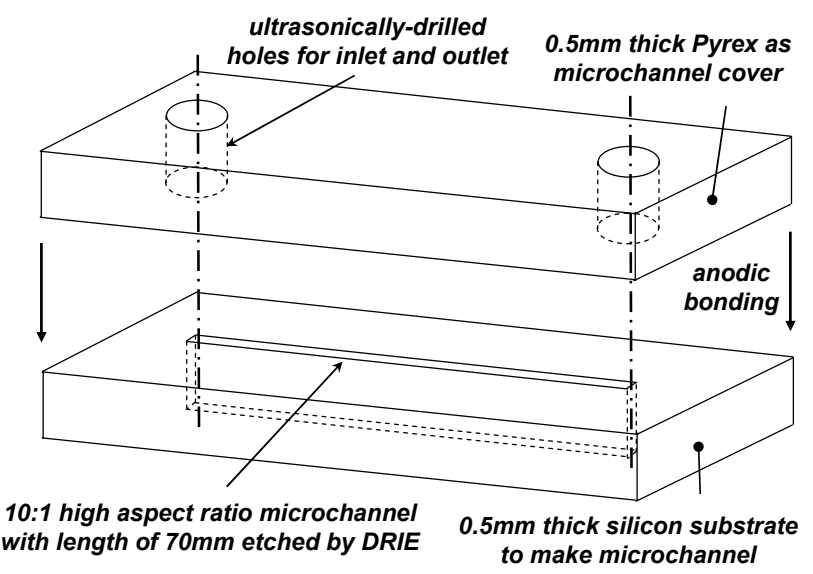

Figure 5: The fabrication and assembly of a microchannel chip.
In the experimental setup, a CCTV camera (TK-C1480E, JVC, Japan) was mounted onto the microscope. The microchannel chip was placed on the stage of the microscope (Axiophot, Zeiss, Germany). The spheres were illuminated by cool light. The PAL video signal, 25 frames per second, of the camera was forwarded to an analog-to-digital converter (ADVC-110, Canopus, Japan) and recorded via IEEE1394 interface in AVI format in Premiere (Premiere Pro, Adobe, U.S.A.). The AVI was later decomposed into TIF files for image processing. With some scripts in Matlab, the position and the velocity of individual sphere was measured as illustrated in Figure 6.
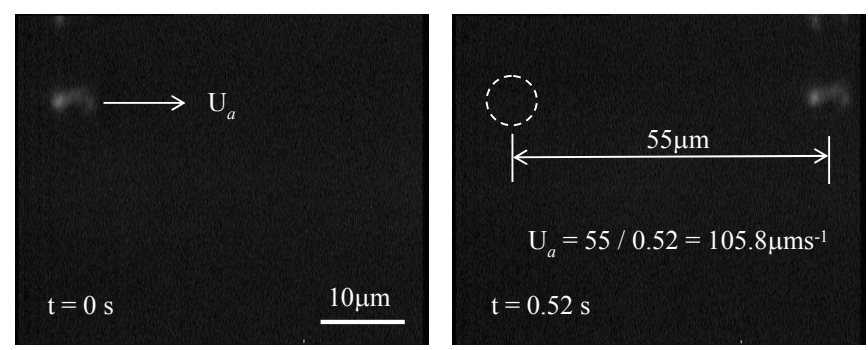

Figure 6: The images of a sphere $(a=2.74 \mu \mathrm{m})$ traveling at

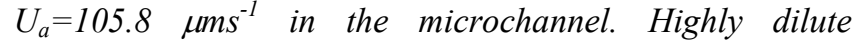
suspensions facilitate the investigation of individual particle-wall interactions.

\section{Experimental Results}

The experimental data are converted into the dimensionless velocity of the sphere $U_{a}^{*}$. It is plotted against the dimensionless channel width, $z^{*}$, as shown in Figure 7.

It shows that the big sphere $(a=2.74 \mu \mathrm{m})$ closest to the wall experiences a significant velocity retardation in comparison to the original pure liquid flow. By contrast, the small sphere $(a=0.91 \mu \mathrm{m})$ follows consistently with the original flow profile, though it is even closer to the wall. To fairly compare the sphere size effect on this velocity retardation, a dimensionless distance $\delta / a\left(=\delta^{*} / a^{*}\right.$ because they are both non-dimensionalized by one-half of the channel height) is proposed to characterize this phenomenon. In Figure 8, we plot the relative velocity deviation against $\delta /$ $a$. The simulation results and the experimental data qualitatively corroborate our physical model in Figure 1 that the decisive factor for the reduction of the particle translational velocity $U_{a}$ is indeed the overlap between the "cloud" and the wall. A measure of this overlap is characterized by the experimentally observable parameter $\delta$ / a. For large distances or small particles, i.e. $\delta / a>>2$, the velocity retardation vanishes. 


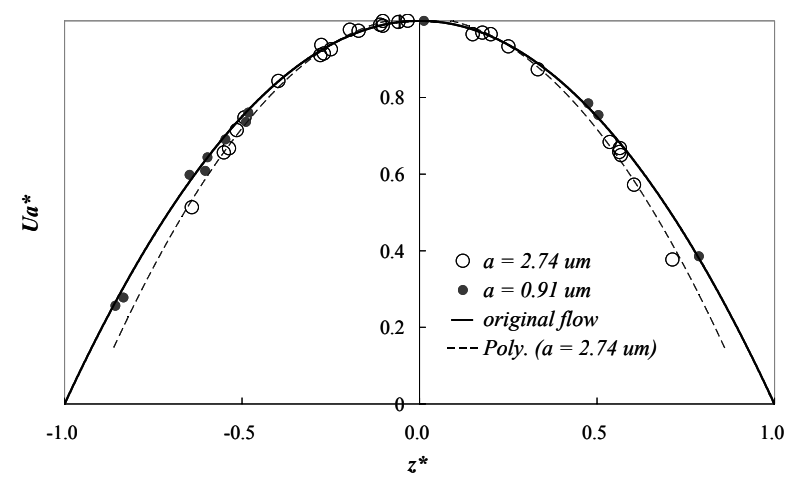

Figure 7: The experimental data of differently sized spheres plotted with dimensionless variables on the axes. Their $U_{a}{ }^{*}$ are compared with the original pure liquid flow profile.

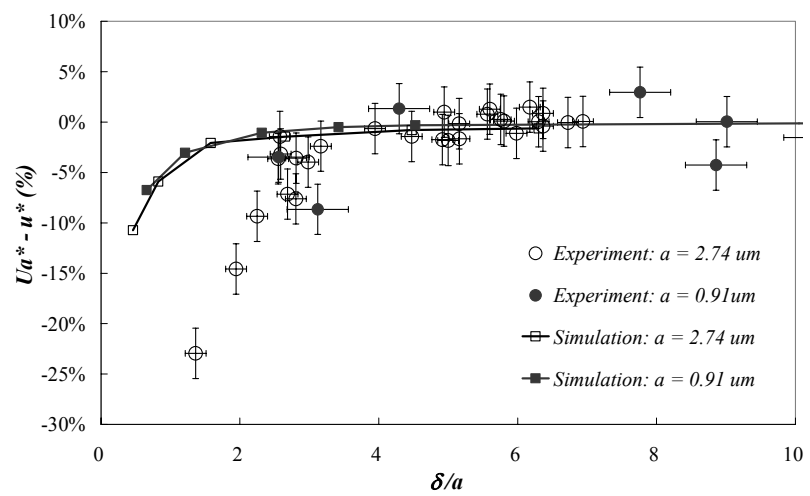

Figure 8: The critical value of $\delta / a$ is around 1 predicted by simulation and is around 2 shown by experiments.

From Figure 8, we find that the velocity retardation measured in experiment was more pronounced than predicted by the simulation model. Such an appreciable quantitative discrepancy will be further investigated in the near future.

One possible explanation is the presence of an electrical double layer (EDL) around the sphere, a negatively charged PS surface, and on the wall, a negatively charged silicon dioxide surface. In a shear flow experiment in another study, it turned out that many PS particles were stuck on another positively charged polymer coating, poly(allylamine hydrochloride) or $\mathrm{PAH}$, while that rarely happened on the silicon dioxide surface [7].

Therefore, it is believed that a significant repulsion force exists between the EDL of PS particle and the EDL of the wall. A similar argument was used to explain the discrepancy between experimental results and theoretical predictions in the HDC experiments [3]. For the thickness of the EDL of the silicon dioxide surface in deionized (DI) water, it was measured to be $305 \mathrm{~nm}$ at $21^{\circ} \mathrm{C}$ in an electrokinetics experiment [8]. Moreover, based on a simple calculation by assuming $18 \mathrm{M} \Omega \mathrm{cm}$ as the resistivity of DI water and $\mathrm{H}^{+}$and $\mathrm{OH}^{-}$as the only ions present, the thickness of the EDL is roughly estimated to be $1 \mu \mathrm{m}$.
In a first order approximation, one may model the EDL around the sphere by assuming an increased effective hydrodynamic radius of the sphere and that on the wall by assuming a decreased channel height. As such, the radius of sphere $a$ increases and the effective gap $\delta$ decreases, thus reducing the ratio $\delta / a$.

All experimental data hence shift to the left-hand side of the graph in Figure 8. In other words, the discrepancy between the experimental data and the simulation data diminishes. Further experimental investigation is required to prove the effect related to the EDL. Nevertheless, the strong particle-wall interaction shown in this study may help to explain the large deviation between the particle velocity and the liquid velocity that were recently reported in an experimental study [9].

\section{CONCLUSION}

We verified by simulations and accompanying experiments that the velocity of individual particles suspended in a PDF in the vicinity of the wall is significantly reduced with respect to the corresponding velocity of a pure liquid. The effect is governed by the contact of the perturbation cloud with the no-slip boundary condition at the wall. The impact parameters are the particle size and its distance from the wall.

Our results are important to understand particle motion through microchannels in general and also to account for secondary effects in chip-based HDC.

\section{ACKNOWLEDGEMENTS}

Dr. Hau is financially supported by a fellowship from The Croucher Foundation in Hong Kong, China.

Dr. Liu is supported by the BMBF project INVMAC.

\section{REFERENCES}

[1] R. F. Probstein, Physicochemical Hydrodynamics: An Introduction, 2003.

[2] E. Chmela et al., Anal. Chem., 74, 3470, 2002.

[3] M. T. Blom et al., Anal. Chem., 75, 6761, 2003.

[4] J. Happel and H. Brenner, Low Reynolds Number Hydrodynamics, 1963.

[5] H. Lamb, Hydrodynamics, 1932.

[6] C. Pozrikidis, Boundary integral and singularity for linearized viscous flow, 1992.

[7] W. L. W. Hau et al., Microm. \& Microeng., 13, 272, 2003.

[8] D. Li, Electrokinetics in Microfluidics, 2004.

[9] X. Xuan and D. Li, Microm. \& Microeng., 16, 62, 2006. 